\title{
INCIDENCIA DE APLICATIVOS DE APRENDIZAJE MÓVIL EN LA DEMANDA DE FORMACIÓN CONTINUA EN ESTUDIANTES DE LA UCSG CURSANDO LOS ÚLTIMOS TRES SEMESTRES
}

\author{
INCIDENCE OF MOBILE LEARNING APPLICATIONS IN THE DEMAND OF \\ CONTINUING EDUCATION IN STUDENTS ENROLLED IN THE LAST THREE \\ SEMESTERS AT UCSG
}

\section{LUIS FERNANDO ALBÁN', PEDRO ANDRÉS CEDEÑO²}

\footnotetext{
Facultad de Especialidades Empresariales, Universidad Católica de Santiago de Guayaquil

luis.alban01@cu.ucsg.edu.ec

2 Facultad de Especialidades Empresariales, Universidad Católica de Santiago de Guayaquil
}

RESUMEN

Esta investigación analizó a los estudiantes que estaban por egresar de la UCSG, para estimar las necesidades de formación continua y utilidad de los aplicativos móviles, nivel de aceptación, requerimientos y necesidades (temáticas y facilidad de uso) en el diseño de aplicativos de aprendizaje, mediante encuestas de gustos y preferencias. Los resultados encontrados hacia el aspecto de dispositivo es que este tiene que ser un smartphone, con Sistema Android, el mismo que tuvo una aceptación del $68 \%$ vs los otros sistemas Symbian 4\%, Blackberry 7\%, IOS $28 \%$. En el aspecto de aprendizaje se encontró que el $40 \%$ si realizan cursos de capacitación 0 actualización con una media de tres cursos al año, los mismos que son realizados en instituciones ajenas a las la UCSG en un $80 \%$; los aplicativos que se utilizaron con mayor frecuencia fueron las redes sociales (80\%), envío y recepción de mensajes de texto (70\%), leer noticias (57\%), investigaciones $(50 \%)$, escuchar música $(65 \%)$ y ver videos $(70 \%)$. Se lograron confirmar las tendencias de comportamiento de los individuos sobre el uso de dispositivos y formas que estos lo utilizan, para de esta manera determinar un marco referencial para la ejecución de la siguiente etapa donde se ejecutarán las conjeturas de las primeras investigaciones y se llevará a cabo el diseño, desarrollo y prueba de un aplicativo de aprendizaje móvil piloto.

PALABRAS CLAVE: educación continua, aplicativos móviles, dispositivos tecnológicos, Smartphone, Android, IOS, SMS, redes sociales, audiobooks.

\begin{abstract}
This research analyzed students enrolled in the last three semesters at UCSG in order to estimate the need for continuous education and usage of mobile applications, level of recognition, requirements (topics and usability) to design the learning applications through surveys based on needs and preferences variables. The results in terms of the aspect of the device showed that $68 \%$ preferred an Android operating system vs. $4 \%$ Symbian, Blackberry 7\%, 28\% IOS systems. The learning aspect results demonstrate that $40 \%$ had taken training courses in an average of three courses per year, more over, $80 \%$ of these courses were taken outside UCSG. The most used application among the participants were social networks (80\%) such as, sending and receiving text messages (70\%) news (57\%), research (50\%), listening to music (65\%) and video (70\%). The research was able to determine the behavior of individuals toward the use of mobile devices; furthermore, these results established a framework for the next stage of the research, which will analyze the relation of the first stage and second stage in order to develop the mobile learning application and its implementation.
\end{abstract}

KEYWORDS: continuing education, mobile applications, technological devices, Smartphone, Android, IOS, SMS, social networks, audiobooks. 


\section{INTRODUCCIÓN}

La forma en que el ser humano se comunica, ha evolucionado y sigue haciéndolo de manera acelerada en estos tiempos, donde la innovación tecnológica es la primicia de los sistemas económicos sustentables para sobrevivir. La educación así mismo debe evolucionar y adaptarse a las nuevas tendencias de comunicación, para capacitar al individuo hacia un verdadero camino de desarrollo profesional, económico y social. Las TIC en la última década han transformado los comportamientos de los consumidores, así como también las metodologías de la enseñanza y la forma en que los estudiantes de la educación superior interactúan dentro y fuera de los salones de clases, espacios virtuales y la forma en que estos se comunican.

En la primera fase de esta investigación se recopiló información y se desarrolló un primer informe pormenorizado de los tipos de dispositivos, diseño de aplicativos móviles, métodos y forma de aprendizaje de estudiantes de universidades españolas y de EE.UU.; el informe sobre los estudiantes egresados, consultados sobre el uso de dispositivos móviles y aplicativos utilizados, confirmaron las tendencias de comportamiento de los individuos sobre el uso de dispositivos y formas que estos lo utilizan.

La propuesta de esta segunda etapa de la investigación es confirmar la necesidad de una herramienta tecnológica a la metodología tradicional de formación, como la educación continua, que cada vez tiene menos adeptos por distintas circunstancias que se describirán en el presente reporte de la investigación. Se llevará a cabo una investigación pormenorizada en el estudiantado de la UCSG, para conocer sus herramientas de esparcimiento y comunicación tecnológica actual, sus necesidades de aprendizaje y su disponibilidad para acceder a dicho aprendizaje.

Este estudio investigativo tiene como objetivo general "Evaluar la incidencia del uso de los aplicativos de aprendizaje móvil en la demanda de educación continua, mediante un estudio dirigido a estudiantes de los tres últimos años de la UCSG, para diseñar un aplicativo móvil”; para poder cumplir este objetivo se realizarán los siguientes objetivos específicos:

1. Estimar las necesidades de formación continua y utilidad de los aplicativos móviles, mediante encuestas de gustos y preferencias a los estudiantes por egresar.
2. Desarrollar una investigación de mercado sobre el nivel de aceptación, requerimientos y necesidades (temáticas y facilidad de uso) en el diseño de aplicativos de aprendizaje para estudiantes por egresar de la UCSG.

\section{METODOLOGÍA}

El modelo referencial para el desarrollo de la correlación con las variables que formulan el presente trabajo de investigación, será el marco referencial para el análisis racional de la educación móvil o FRAME (Framework for the Rational Analysis of Mobile Education)(Koole, 2006).

El modelo FRAME, considera tanto los aspectos técnicos de los dispositivos móviles, así como el aspecto social y personal del aprendizaje. En base a este modelo se desarrolló una serie de preguntas directas y con varias connotaciones en formato de cuestionario, para la exploración de cada uno de estos aspectos.

Se estableció como una muestra considerable para el universo de estudiantes de la universidad católica trabajar con 334 personas elegidas aleatoriamente; este fue el número de encuestas llevadas a cabo a los estudiantes de UCSG que se hallaban cursando los últimos módulos de sus carreras, los cuales transcurren en la mayoría de carreras en el módulo sexto hasta noveno. Estudiantes del séptimo módulo de su carrera fueron la mayoría, con un $60 \%$ de la muestra, seguido por los de octavo con un $20 \%, 15 \%$ por los de noveno y por último los de sexto con un $5 \%$ de participación.

Se tuvo una aceptable participación por parte de ellos, y se los identificó de 14 carreras de las nueve facultades de la UCSC, donde predominaron las participaciones de la carrera de derecho, seguida de ingeniería civil, filosofía, medicina, arquitectura y multimedia. Además hubo así mismo una superior participación por parte de estudiantes de sexo femenino con un $57 \%$, versus un $43 \%$ de participación de público masculino; la gráfica contigua muestra el detalle de los entrevistados por carrera y sexo de los entrevistados.

\section{RESULTADOS}

La primera pregunta iba enfocada a que la persona encuestada defina el nivel de frecuencia con que utilizan el computador de escritorio, la laptop, tabletas y celulares; el celular supera un $90 \%$ su utilización de frecuencia, y lo convierte en el dispositivo más utilizado de todos, superando con creces la laptop con $60 \%$ de frecuencia 
de uso, al computador de escritorio con el $45 \%$, y a la Tablet con un $32 \%$; este último dispositivo adolece de uso más frecuente, por asuntos de practicidad, ya que la tableta sirve más para consumo que para producción de contenido, para lo cual este último es más importante al estudiante universitario que se ve día a día investigando y generando reportes para sus materias, ya que al analizar las respuestas por carreras se aprecia que más del $75 \%$ de los encuestados de Medicina tienen un uso intensivo de la tableta, ya que su labor se centra en la lectura intensiva de textos, seguida por Filosofía y Derecho. Esta pregunta es el primer pilar de la investigación hacia la relevancia del enfoque que debe llevar el desarrollo de un aplicativo más hacia un celular que hacia otro de los dispositivos en mención, al ser revelado como el de mayor frecuencia de utilización.

Los celulares se dividen en dos al menos en términos de diseño de aplicativos que interactúen con el usuario final, ya que modelos básicos de celulares no tienen plataformas estandarizadas ni pantallas adecuadas para diseñar aplicativos, mientras que los celulares inteligentes están definidos en ciertas plataformas ya universalmente estandarizadas.

Se preguntó si tenían Smartphone o celular inteligente, con un $95 \%$ de confirmaciones. Paso seguido se procedió a preguntar el sistema operativo que utilizaba el celular, donde un notorio $60 \%$ identificó su dispositivo con el sistema Android (incluidos los que no tuvieron Smartphone pero si Tableta, la cual llega apenas al $2 \%$, por lo que es marginal la distinción entre dispositivos), seguido por el IOS de Apple con un $28 \%$, Blackberry OS con un $7.4 \%$ y Nokia compartida con sus dos sistemas vigentes, Windows y Symbia, con un 4\% aproximadamente.

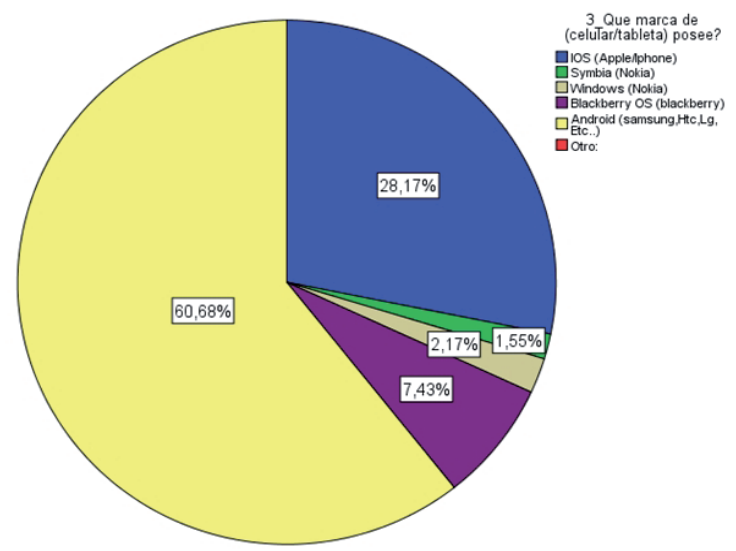

Figura 1. Distribución de sistemas operativos en los dispositivos móviles de los sujetos investigados
El cuarto lugar de preferencia, Nokia, al ser absorbido por Microsoft (Microsoft, 2013), irá paulatinamente desplazando al sistema inicial Symbia, y esto ya se ve a breves rasgos en los números obtenidos, en donde la posesión de dispositivos Nokia con sistema Windows Mobile integrado es levemente mayor que el anterior; pese a que el Blackberry es el tercer dispositivo en participación con $7.4 \%$, que maneja un sistema con su mismo nombre, se destaca que es una tecnología que se vislumbra en pronto desuso por agravantes financieros en los últimos años, modelos de teléfonos que no han sido exitosos y anuncios de la misma compañía que para el 2014 dejaría de fabricar teléfonos para dedicarse al software y servicios para empresas, mensajería, QNX y sistemas empotrados, y terminales (Velasco, 2013). Esta participación se debe redistribuir en los primeros tres sistemas mencionados.

La definición de la plataforma más utilizada es muy importante porque permite proponer el desarrollo del aplicativo dedicado al sistema más común entre los usuarios de teléfonos inteligentes, y que en esta primera etapa de la investigación desarrollada con estudiantes egresados de la UCSC, demuestra que debe ser considerada basarse en el sistema Android, seguida por IOS, ya que ambas acaparan casi el $90 \%$ de preferencias de dispositivos móviles inteligentes.

Las preguntas referentes a los usos o actividades más o menos frecuentes ejecutadas en los smartphones, se pudo cuantificar que más del $70 \%$ de usuarios no lo utilizan o es muy poco frecuente para videollamadas, jugar en un 60\% de desuso así como la lectura de libros (inadecuada lectura extensiva por el tamaño de los dispositivos) con más el $65 \%$ en su ausencia total o parcial de utilización con este propósito.

Audiobooks es una de las actividades con menor aceptación entre estudiantes, con más de $3 / 4$ partes que lo definieron de muy poca utilización en sus dispositivos; este formato de consumo literario es muy arraigado en la cultura norteamericana y de Reino Unido, ya que están estrechamente relacionados al walkman, la radio, el lápiz memoria y la falta de tiempo disponible para la lectura de acuerdo con Vallorini (2011), mientras que en países de habla hispana no es parte de sus costumbres la lectura en formato oral - digital.

El desarrollo de tareas a través del teléfono móvil, denota un uso moderado, al concentrarse la mayor cantidad de respuestas en niveles 
intermedios con un $60 \%$ de los datos entre usos de frecuencia moderada y usos frecuentes; por carreras se encontró una preferencia un poco mayor en Economía, Arquitectura, Derecho y Filosofía, con un poco más de la mitad de los encuestados por carreras afirmando una mayor frecuencia en la ejecución de tareas a través del dispositivo; temas como formación, educación, preparación, arrojan resultados con tendencia moderada de uso, similar al desarrollo de tareas en el móvil, con igual $60 \%$ de frecuencia, y con un marcado $27 \%$ de inutilidad para este propósito; estos resultados pueden deberse al desconocimiento o carencia de aplicativos propios desarrollados por la universidad y ajustados a las necesidades de los estudiantes; analizada por carreras, tampoco se halla una marcada tendencia en el uso del dispositivo para su autoeducación.

Las preguntas que marcaron clara tendencia en todos los encuestados por su uso intensivo en celulares inteligentes, fueron las redes sociales $(80 \%)$, envío y recepción de mensajes de texto $(70 \%)$, leer noticias $(57 \%)$, investigaciones $(50 \%)$, escuchar música $(65 \%)$ y ver videos (70\%) cuyas dos últimas preguntas previendo estos resultados se llevaron a cabouna serie de preguntas adicionales para que el encuestado defina las características de extensión de los videos y música escuchada.

En la gráfica contigua se consideran solo los que evaluaron las características de las lecturas que realizan en su dispositivo móvil con más del $50 \%$ de contestaciones. En general, indistinto de la frecuencia de lectura, son los textos cortos que predominan en el celular; sin embargo es destacable que los de mayor frecuencia de uso, son los que consideran la lectura de textos más extensos. Analizando estos resultados desde cada carrera, se halló que en las de Derecho, Medicina y Filosofía predominan las lecturas largas y cortas, más que en las otras carreras.

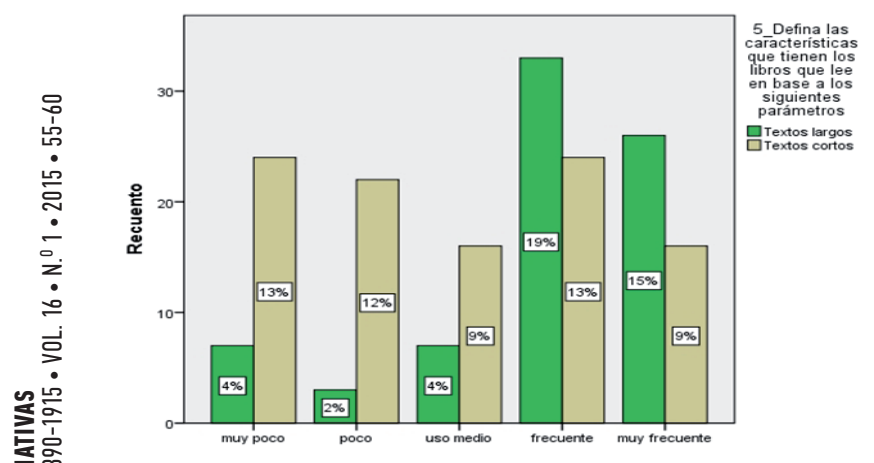

Figura 2. Frecuencia del tipo de lectura en dispositivos móviles.
Las preguntas relacionadas a los tipos de videos, visualizados en el celular y contestados por aproximadamente $2 / 3$ de los encuestados, denotaron una mayor exposición a videos que no están relacionados con la carrera, así mismo como la frecuencia más elevada son los de corta duración; este tipo de videos son los que se visualizan en redes sociales y canales de videos como el YouTube, en donde básicamente se ven videos de entretenimiento y ocio; analizando las dos respuestas indistinta de su relación con la carrera, predominan los videos de igual manera de corta duración, lo que vislumbra y asevera la duración del contenido audiovisual que se deberá desarrollar para el aprendizaje móvil.

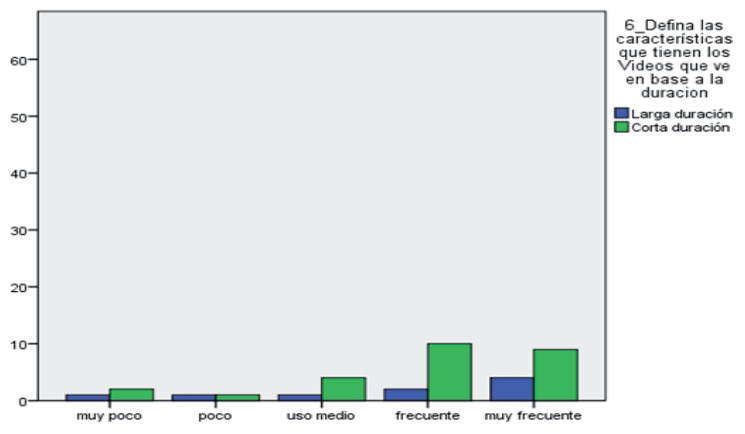

Figura 3. Frecuencia de la durabilidad del contenido audiovisual observado en dispositivos móviles, y relacionado con sus estudios.

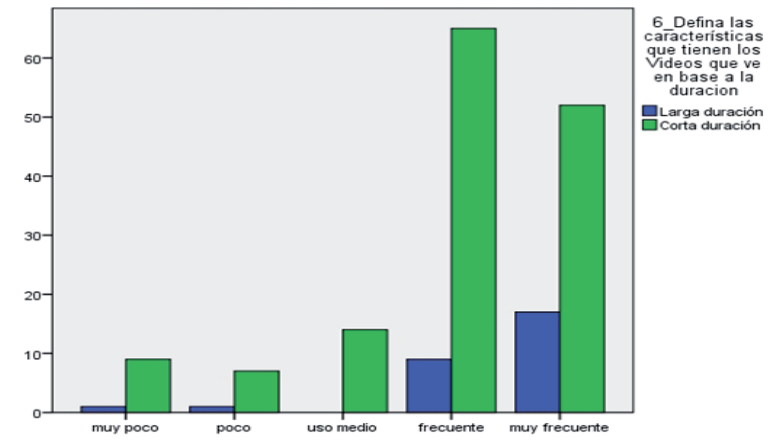

Figura 4. Frecuencia de la durabilidad del contenido audiovisual observado en dispositivos móviles, y que no tienen relación con sus estudios.

Con respecto a la pregunta complementaria a la actividad de escuchar música, menos del $2 \%$ escucha radio por Computador o Laptop, $25 \%$ lo escucha a través del celular ya sea por aplicativos radiales en sus dispositivos o música almacenada dentro del mismo, y casi el $40 \%$ en el auto; apenas el $9 \%$ de todos los encuestados participan con el locutor de radio a través de redes sociales o llamando directamente para participar en alguna actividad propuesta por 
la radio; pese que la actividad de escuchar música tiene una frecuencia significativa, su nivel de participación es bajo, denotando que el desarrollo de actividades instruidas por voz para el aplicativo de aprendizaje, no es viable. Esta primera parte brindó las primeras pautas para el desarrollo de contenido y entornos de difusión más adecuados para el aplicativo que se pretende diseñar.

Posteriormente se cuestionó sobre la capacitación de los estudiantes, en que un $40 \%$ aproximadamente confirmaron que habían asistido a un curso de capacitación reciente; solo de este número de estudiantes se prosiguió preguntando cuantas veces al año había participado en cursos de capacitación obteniendo los siguientes resultados: en promedio las personas realizan tres capacitaciones al año con una concentración de más del $88 \%$ entre uno y cinco capacitaciones al año; paso seguido se procedió a preguntar si estas capacitaciones eran llevadas a cabo en la UCSG, de los cuales menos del $20 \%$ vuelven a la universidad para hacer estos cursos de actualización, y de éstos el $80 \%$ ha cogido hasta tres cursos en la UCSC durante el último año.

Casi un 34\% de los encuestados le dan una preferencia al desarrollo del aplicativo desarrollado únicamente al celular, y más del 76\% un uso compartido entre celular, tableta y PC, lo cual ratifica la gestión del desarrollo de un aplicativo para estos dispositivos, de preferencia en el celular que es el de mayor aceptación, accesibilidad, portabilidad y finalidad del aprendizaje móvil en sí.

La finalidad de la pregunta sobre la frecuencia de utilización de las redes sociales, es para conocer el medio de comunicación, interacción y promoción del aplicativo más adecuado, además de definir ciertas características adicionales de los potenciales usuarios del aplicativo, de acuerdo a la red social usada con mayor frecuencia; se requirió al encuestado que determine la frecuencia de uso de cada red social, cuyos resultados dan a la red Facebook con casi el 67\% de utilización de mayor frecuencia, seguido muy de cerca por Twitter y Google + con el $65 \%$ de uso frecuente, y la red Istagram, con el 50\% de mayor frecuencia de utilización; otras redes sociales que identificaron los estudiantes, fueron Snapchat, Flicker, Hi5, Linkedin, Pinterest, Tumblr, y Apps de comunicación como Skype, Whatsapp y Line, las cuales en su conjunto no llegan al 9\% de la muestra, por lo que su consideración es marginal, y ratifica el posicionamiento de las cuatro primeras redes en las que se identificaron la gran mayoría de individuos.

\section{DISCUSIÓN}

Al tener claramente dos marcadas tendencias de sistemas en los dispositivos móviles, IOS y Android, se debería desarrollar la compatibilidad del aplicativo de acuerdo al uso más frecuente, identificado por carrera, ya que se aprecia unas leves tendencias de mayor preferencia para ciertos dispositivos.

Cuando se analizó la capacitación de los estudiantes, si bien dejó una buena impresión por el interés en mantenerse al día, surgió en relación a esta pregunta, la sugerencia de estudiar mejor las razones por las que este amplio público no participa en las capacitaciones ofrecidas por la misma universidad, pese a que aún se encuentran activos en la misma.

\section{CONCLUSIONES}

El tópico M-learning es uno de los temas candentes en la literatura que aborda cuestiones sobre nuevas tecnologías en educación. Más aún cuando autores como Rius, Clarisó, y Masip (2014) mencionan que vivimos en la que se ha denominado la era móvil, en lo que existe una revolución tecnológica protagonizada por los dispositivos móviles.

Este segundo reporte señaló que los estudiantes encuestados, en su mayoría mujeres $57 \%$, interactúan muy poco con el dispositivo móvil "Tableta” pero si muestran un alto grado de interacción con su dispositivo móvil "Celular Inteligente”; así también el software de mayor uso dentro de esta institución de educación superior es Androide con una aceptación del $68.68 \%$ seguido de IOS con $28.17 \%$. En el siguiente campo de indagación sobre capacitación profesional se encontró que los estudiantes de los últimos tres ciclos de las 14 carreras encuestadas realizan cursos de capacitación con un aproximado de $40 \%$ y solo el $20 \%$ elige la UCSC para capacitarse. Finalmente, este estudio confirma que los estudiantes poseen un favoritismo hacia el desarrollo de este aplicativo de educación continua para sus "Celulares Inteligentes" y que sea promovido a través de la red social Facebook y Twitter.

AGRADECIMIENTOS 
Agradecemos al SINDE y al SED por brindarnos su apoyo constante en la capacitación, injerencia para la prestación de la base de datos de la universidad, y el cuerpo docente integral de estas unidades que siempre apoyaron para que

\section{REFERENCIAS BIBLIOGRÁFICAS}

Bao, Yan (2008). A study on the personal and social environment influencing working youth's participation in continuing education programs in Shanghai. Hong Kong. http://hdl. handle.net/10722/54458

Cataldi, Z., Méndez, P., Dominighini C., Lage, F. (2012). Dispositivos móviles en educación superior y entornos personalizados de aprendizaje. http://hdl.handle.net/10915/19437

Ceccaroni, L., Willmott, S., Cortés, U., BarberaMedina, W. (2005). @LISTechNET: Demostración de tecnología avanzada para educación y aplicaciones culturales en Europa y América Latina. http://www.lsi.upc.edu/ luigi/papers/ Ingenieria_al_dia-2005.pdf

Diario Expreso (5 de Febrero de 2014). Smartphone el equipo que nos cambió la vida. Recuperado de: http://expreso.ec/expreso/plantillas/ nota_print.aspx?idArt $=5697286 \&$ tipo $=2$

El financiero (6 de Marzo de 2014). El futuro de BlackBerry suena mejor sin smartphones. Recuperado de: http://www.elfinanciero.com. mx/archivo/el-futuro-de-blackberry-suenamejor-sin-smartphones.html

ISEA S.Coop. (2005). MOBILE LEARNING, Análisis prospectivo de las potencialidades asociadas al Mobile Learning.

Koole, M. (2006). Framework for the rational analysis of mobile education (FRAME): A model for evaluating mobile learning devices. Thesis, Centrefor Distance Education, Athabasca University. no desmaye el desarrollo de esta investigación; concretamente al Dr. Castaño y la Lcda. Reyes, puntales de estos centros de generación y conocimiento de la universidad y la sociedad.

Microsoft News Center (3 de septiembre de 2013). Microsoft to acquire Nokia's devices \& services business, license Nokia's patents and mapping services. Recuperado de: http://www.microsoft.com/en-us/news/press/2013/sep13/0902announcementpr.aspx

Mohamed, A. (2009). Mobile Learning: Transforming the Delivery of Education and Training. Collette, Québec

Planell, J. (2014). Las universidades serán 'online' en el año 2050. Recuperado de: http:// www.eluniverso.com/vida-estilo/2014/02/02/ nota/2120306/universidades-seran-onlineano-2050\}

Rius, A., Clarisó, R., Masip, D. (2014). Student projects empowering mobile learning in higher education. http://dx.doi.org/10.7238/rusc. v11i1.1901

Vallorani, C. (2011). La oralidad tecnológica-digital: estudio pragmático-comunicativo sobre la oralidad en el audiolibro. http://hdl.handle. net/10045/19656

Velasco, J.J. (30 de Septiembre del 2013). ¿Cuál será la estrategia de BlackBerry para el año 2014?. Recuperado de: http://hipertextual. com/2013/12/estrategia-blackberry-2014 